\section{Virus genetic variability involvement in transmissibility of HIV-1 immune activation and disease progression}
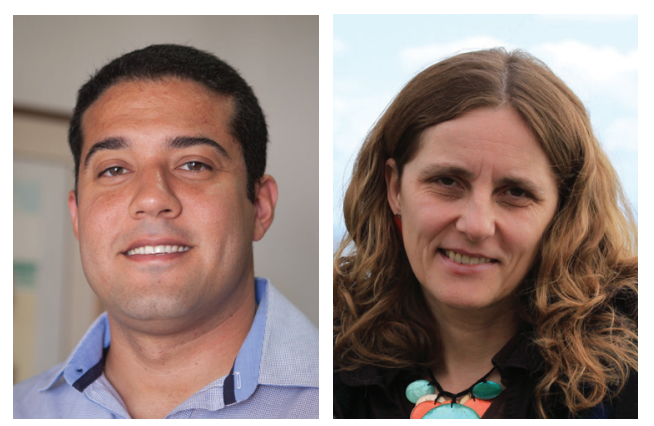

\author{
“The high viral replicative \\ capacity associated with \\ sustained and uncontrolled \\ immune activation makes \\ aggressive HIV-1 variants a real \\ threat to the control of the \\ global epidemic of HIV-1.”
}

Ricardo Khouri',2 \& Anne-Mieke Vandamme ${ }^{*, 2,3}$

For decades, immunologists and virologists have worked somewhat independently trying to understand AIDS disease progression for the benefit of improved patient care. Their efforts have resulted in the development of antiretroviral therapy (ART) as well as contributed to our understanding of immunological factors associated with disease progression. Disease progression occurs when virus replication is not sufficiently controlled, which is observed when HIV evades selective pressure imposed by the immune system and/or antiviral drugs. The high evolutionary rate of HIV allows for continuous adaptation of the virus to different intrahost pressures (e.g., ART and immune response). We are starting to understand how the selection processes could optimize virus fitness and how the resulting changes in the virus can be transmitted.

Throughout the asymptomatic phase of HIV-1 infection in ART-naive patients, the viral load is kept at relatively stable levels over several years, known as the set point viral load (SPVL). This is a dynamic process, in which some viruses are continuously adapting and escaping the immune selective pressure. It is well documented that SPVL levels can differ substantially between patients, and that a high SPVL value is one of the most important predictors of fast disease progression [1]. Fraser et al. found that high SPVL can be transmitted to newly infected patients and suggested that SPVL values may result from determinants of the virus itself and/or host factors such as immune activation profile or specific host genetic variants. Thus, SPVL reflects a complex interplay between the immune responses in the newly infected host and the replication capacity of the virus [2]. One important unresolved issue is the identification of the genetic region of the virus that may be the key determinant of the SPVL transmissibility. Recently, HIV/AIDS researchers have started to combine the knowledge of

\section{KEYWORDS}

- AIDS • coreceptor • CRFs fitness

- CXCR4 - disease progression

- immune activation • protease

- RANTES • transmission

"Only the understanding of the fine-tuning between virus replicative mechanisms and immune activation

might put a damper on disease progression..." 
virus evolution and that of disease progression, which has resulted in increasing numbers of publications that improve our understanding of the causes of HIV-1 genetic variability, SPVL transmissibility and faster disease progression.

The observation that syncytium-inducing viruses were strongly linked to HIV-1-driven disease progression was one of the first discoveries on how HIV-1 genetic variability could affect the clinical prognosis of infected patients [3]. Further studies discovered that the capacity to induce syncytia was linked to genetic variability in the $e n v$ region and to the capacity of the virus to bind the coreceptor CXCR4. Most HIV-1 infections initially use the CCR5 coreceptor for entry into host cells and switch to use the CXCR4 coreceptor only after several years. Interestingly, the switch to the use of the CXCR4 coreceptor for HIV-1 infection has been previously associated with accelerated progression to AIDS. Thus, it is reasonable to speculate that the env genetic region of HIV-1 is one of the determining factors driving increased risk for accelerated disease progression in infected individuals.

Theys et al. recently demonstrated that most of the protease polymorphisms involved in improving viral fitness in the presence of treatment or resistance mutations, are also contributing to a higher viral load in therapy naive patients without resistance mutations $[4,5]$, most probably by improving enzymatic efficiency. This is in keeping with the fact that the same polymorphic positions also contribute to the replication capacity of the virus $[4,5]$. These observations introduced for the first time the possibility that treatment could contribute to an increase in disease progression at the population level, through transmission of such fitness-related polymorphisms in proteases. Moreover, such findings suggest that $P R O$ may be an important genetic region contributing to SPVL.

It is a great opportunity to study viral genes involved in transmissibility of disease progression when epidemics with more than one HIV-1 subtype overlap within a population, leading to an increased prevalence of recombinants of two distinct virus subtypes (A-K). This is because there are differences in disease progression among subtypes. For example, subtype D infected patients, on an average, progress faster to AIDS than subtype A infected patients [6]. Many of these newly recombined strains do not survive for long. However, recombination between subtypes may give rise to more pathogenic strains if genomic fragments from these different subtypes join together in a virus achieving a higher SPVL. Furthermore, in such epidemiologic scenarios, it is reasonable to expect that only recombinant viruses with the best evolutionary fitness would tend to survive in the affected population, and become circulating recombinant forms (CRFs). This supports the idea that recombination may be an attractive way by which HIV-1 increases overall fitness in a population of infected individuals. This fact does not necessarily imply that virus recombination affects disease progression in consecutive transmissions at a population level. However, more aggressive forms of HIV-1 have been reported in some CRFs, such as the recently examined CRF19_cpx [7], CRF02/A3 [8] and CRF14_BG [9]. Additional studies on CRFs in different populations could clarify whether single or combined genes are the key drivers of accelerated disease progression in a newly infected individual.

A recent report on an aggressive CRF sheds light on the understanding of the HIV-1 genetic region, which may be responsible for transmissibility of accelerated disease progression. Kouri et al. [7] examined the aggressive CRF19_cpx, which is found in epidemic proportions only in Cuba. The investigators described how this virus replicates to achieve very high viral loads, leading to progression to AIDS within 3 years after initial infection. This dramatically accelerated disease progression indicates that CRF19_cpx may be the most aggressive CRF detected so far. CRF19_cpx is a recombinant form of the HIV-1 subtypes A, D and G. Interestingly, CRF19_cpx is subtype D, with regards to its protease, the subtype associated to fast disease progression [6]. This protease has been shown by Kouri et al. to have a very high in silico estimated fitness. CRF19_cpx is subtype A with regards to the envelope, the subtype associated to slower disease progression [6], questioning the role of $e n v$ in the transmissibility of disease progression. CRF19_cpx uses the CXCR4 coreceptor early on for its entry mechanism into the patient's cells which is consistent with fast (rapid) disease progression [7], whereas subtype $\mathrm{A}$ is known to mainly use CCR5. Higher levels of chemokine RANTES were found in CRF19_cpx infected patients and this was correlated with higher viral load $[7,10]$. A protective role of inflammatory CCR5-ligands, namely chemokines RANTES, MIp-1b and MIP-1a, in elite controllers has been 
shown against CCR5 coreceptor using variants, but not for CXCR4 using variants [11], as these chemokines are the natural ligands of CCR5 and block virus entry through the CCR5 coreceptor. Bayesian network analysis allowed investigation of the relationship between the variables and together with other information Kouri et al. hypothesized that a fit protease may contribute to a very high viral load to which the body reacts with high RANTES levels. The use of CXCR 4 as coreceptor by CRF19_cpx might be a virus escape mechanism against high chemokine RANTES production by the host, driving faster disease progression. This paper would thus also support $P R O$ as a contributing gene to the transmissibility of disease progression.

In another publication this year, the relationship between HIV-1 replicative capacity, gag sequence, immune activation and disease progression was investigated [12]. The authors demonstrated that patients infected with HIV-1 variants with gag sequences that contributed to a higher replicative capacity had a significantly higher immune activation and a faster drop of CD4 levels than patients infected with HIV-1 variants with a lower replicative capacity. While the focus here is on Gag [12], it would be interesting to also verify the fitness of protease in these patients, since it is known that Gag and protease are coevolving [13].

While high HIV-1 replication is known to exhaust the immune response, coinfections exacerbate this by starting a vicious cycle of induced high HIV-1 replication, immune response exhaustion, further coinfections and faster disease progression. In the study by Kouri et al. [7] oral candidiasis coinfection was identified as a risk factor for fast progression associated only with CRF19_cpx infection. This vicious cycle mechanism of coinfection and virus replication has been confirmed recently in a study of 717 untreated participants led by the SWISS

\section{References}

1 De Wolf F, Spijkerman I, Schellekens PT et al. AIDS prognosis based on HIV-1 RNA, $\mathrm{CD} 4^{+} \mathrm{T}$-cell count and function: markers with reciprocal predictive value over time after seroconversion. AIDS 11(15), 1799-1806 (1997).

2 Fraser C, Lythgoe K, Leventhal GE et al. Virulence and pathogenesis of HIV-1 infection: an evolutionary perspective. Science 343(6177), 1243727 (2014).

cohort [14]. The authors demonstrated that sCD14 (a marker of LPS bioactivity) is significantly associated with both I-FABP (a marker of gastrointestinal disruption) and viral load, reinforcing the role of microbial translocation in the pathogenesis of untreated HIV-1 infection.

HIV-1 is considered a major public health problem. While antiretroviral therapy contributes significantly to keeping infected individuals in a healthy state, a considerable percentage of patients still progress to AIDS. The high viral replicative capacity associated with sustained and uncontrolled immune activation makes aggressive HIV-1 variants a real threat to the control of the global epidemic of HIV-1. The rapid impairment of the infected individual's health when infected with an aggressive virus can put patients at risk of death even before they are aware that they are infected. Here we summarized the potential role of various HIV-1 genes ( $\mathrm{gag}$, pol and env) in disease progression and its transmissibility, strengthening the need of future viral whole-genome studies associated with a systems immunology approach of the host. Only the understanding of the fine-tuning between virus replicative mechanisms and immune activation might put a damper on disease progression, which does occur albeit slowly even in HAART responders.

\section{Financial \& competing interests disclosure}

This work was supported in part by 'Programa Ciências Sem Fronteiras' Conselho Nacional de Desenvolvimento Cientifico e Tecnológico (CNPq) of Brazil, and Fonds voor Wetenschappelijk Onderzoek Vlaanderen (G.0692.14). $R$ Khouri was supported by an IRO scholarship-KU Leuven. The authors have no other relevant affiliations or financial involvement with any organization or entity with a financial interest in or financial conflict with the subject matter or materials discussed in the manuscript apart from those disclosed.

No writing assistance was utilized in the production of this manuscript.

3 Richman DD, Bozzette SA. The impact of the syncytium-inducing phenotype of human immunodeficiency virus on disease progression. J. Infect. Dis. 169(5), 968-974 (1994).

4 Theys K, Abecasis AB, Vandamme AM. HIV-1 drug resistance: where do polymorphisms fit in? Future Microbiol. 8(3), 303-306 (2013).

5 Theys K, Deforche K, Vercauteren J et al. Treatment-associated polymorphisms in protease are significantly associated with higher viral load and lower CD4 count in newly diagnosed drug-naive HIV-1 infected patients. Retrovirology 9, 81 (2012).

6 Baeten JM, Chohan B, Lavreys L et al. HIV-1 subtype $\mathrm{D}$ infection is associated with faster disease progression than subtype $\mathrm{A}$ in spite of similar plasma HIV-1 loads. J. Infect. Dis. 195(8), 1177-1180 (2007).

7 Kouri V, Khouri R, Alemán Y et al. CRF19_cpx is an evolutionary fit HIV-1 variant strongly associated with rapid progression to AIDS in Cuba. EBioMedicine 2(3), 244-254 (2015). 
8 Palm AA, Esbjörnsson J, Månsson F et al. Faster progression to AIDS and AIDS-related death among seroincident individuals infected with recombinant HIV-1 A3/CRF02_AG compared with sub-subtype A3. J. Infect. Dis. 209(5), 721-728 (2014).

9 Pérez-Álvarez L, Delgado E, Vega Y et al. Predominance of CXCR4 tropism in HIV-1 CRF14_BG strains from newly diagnosed infections. J. Antimicrob. Chemother. 69(1), 246-253 (2014).
10 Loke P, Favre D, Hunt PW et al. Correlating cellular and molecular signatures of mucosal immunity that distinguish HIV controllers from noncontrollers. Blood 115(15), e20-e32 (2010).

11 Walker WE, Kurscheid S, Joshi S et al. Increased Levels of macrophage inflammatory proteins result in resistance to $\mathrm{R} 5$-Tropic HIV-1 in a subset of elite controllers. J. Virol. 89(10), 5502-5514 (2015).

12 Claiborne DT, Prince JL, Scully E et al. Replicative fitness of transmitted HIV-1 drives acute immune activation, proviral load in memory $\mathrm{CD}^{+}{ }^{+} \mathrm{T}$ cells, and disease progression. Proc. Natl Acad. Sci. USA 112 (1), e1489 (2015).

13 Li G, Theys K, Verheyen J et al. A new ensemble coevolution system for detecting HIV-1 protein coevolution. Biol. Direct. 10, 1 (2015).

14 Perkins MR, Bartha I, Timmer JK et al. The interplay between host genetic variation, viral replication, and microbial translocation in untreated HIV-infected individuals. J. Infect. Dis. 212(4), 578-584 (2015). 